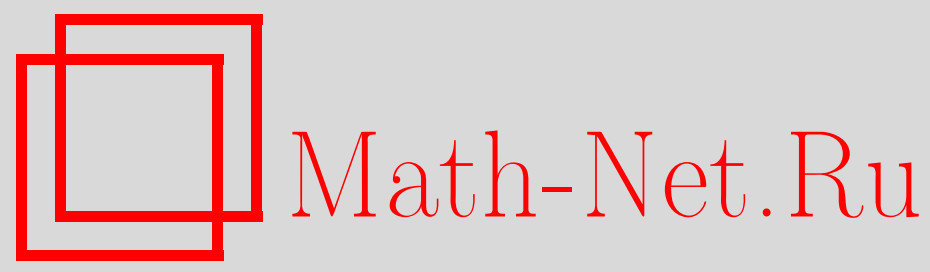

В. П. Бурский, Е. А. Буряченко, Некоторые вопросы нетривиальной разрешимости однородной задачи Дирихле для линейных уравнений произвольного четного порядка в круге, Матем. заметки, 2005, том 77, выпуск 4, 498-508

DOI: https://doi.org/10.4213/mzm2508

Использование Общероссийского математического портала Math-Net.Ru подразумевает, что вы прочитали и согласны с пользовательским соглашением http://www.mathnet.ru/rus/agreement

Параметры загрузки:

IP: 34.239 .49 .27

26 апреля 2023 г., 07:42:34

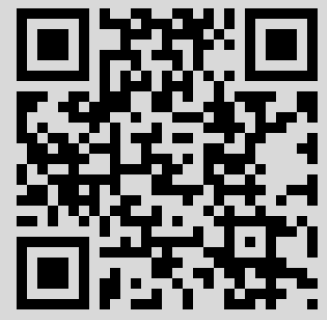




\title{
НЕКОТОРЫЕ ВОПРОСЫ НЕТРИВИАЛЬНОЙ РАЗРЕШИМОСТИ ОДНОРОДНОЙ ЗАДАЧИ ДИРИХЛЕ ДЛЯ ЛИНЕЙНЫХ УРАВНЕНИЙ ПРОИЗВОЛЬНОГО ЧЕТНОГО ПОРЯДКА В КРУГЕ
}

\author{
В. П. Бурский, Е. А. Буряченко
}

\begin{abstract}
В настоящей работе получено необходимое и достаточное условие нетривиалной разрешимости однородной задачи Дирихле в круге для линейных уравнений произвольного четного порядка $2 m$ с постоянными комплексными коэффициентами и однородным невырожденным символом в общем положении. Отдельно рассмотрены случаи $m=1,2,3$. Для случая $m=2$ приведены примеры вещественных эллиптических систем, сводящихся к одиночньм уравнениям с постоянными комплексными коэффициентами, для которых однородная задача Дирихле в круге имеет счетный набор линейно независимых полиномиальных решений.

Библиографофия: 15 названий.
\end{abstract}

1. Введение. Целью настоящей работы является получение критерия нетривиальной разрешимости однородной задачи Дирихле в круге для общего уравнения главного типа четного порядка $2 m$ с постоянными комплексными коэффициентами и однородным невырожденным символом. Отдельно рассматриваются случаи $m=1,2,3$. Эффект нетривиальной разрешимости однородной задачи Дирихле для эллиптических уравнений второго порядка был впервые обнаружен А. В. Бищадзе, который в работе [1] указал пример уравнения, для которого однородная задача Дирихле в круге имеет счетное число линейно независимых решений. Это уравнение имеет вид: $\partial^{2} u / \partial \bar{z}^{2}=0$. Нетрудно видеть, что функция $(z \bar{z}-1) P(z)$ с произвольным полиномом $P(z) \not \equiv 0$ является нетривиальным решением задачи Дирихле для этого уравнения в круге. Затем им же [2] был обнаружен другой пример уравнения второго порядка с постоянными комплексньми коэффициентами, имеющий то же свойство. Уравнения, приведенные Бицадзе, не являются правильно эллиптическими, поэтому для них возможна неединственность решения задачи Дирихле. Напомним, что для правильно эллиптических уравнений четного порядка с постоянньми комплексньми коэффициентами однородная задача Дирихле имеет не более, чем конечное число линейно независимых решений [3], а для таких уравнений второго порядка без младших членов в плоской области имеет место единственность решения [4]. Последнее утверждение является следствием результатов работы М. Й. Вишика [5] о единственности решения задачи Дирихле для уравнений с постоянными комплексньми коэффициентами без младших членов, сводяшихся к вещественньм сильно эллиптическим системам. В работе Р. А. Александряна [6] (см. 
также [7]) было получено условие единственности решения задачи Дирихле в единичном круге для одного гиперболического уравнения в случае, когда $\varphi_{1}=-\varphi_{2}$, где $\varphi_{i}$, $i=1,2,-$ углы наклона характеристик (см. определение в п. 2). Это условие - суть условие иррациональности угла $2 \varphi_{1} / \pi$. Позже, в работе [8], одним из авторов было получено необходимое и достаточное условие нетривиальной разрешимости однородной задачи Дирихле в единичном круге для эллиптических уравнений второго порядка с постоянньпи комплексными коэффициентами и однородным невырожденньп символом в виде $\pi$-рациональности угла между комплексными характеристиками.

Вопросам единственности решения задачи Дирихле в круге для правильно эллиптических уравнений четвертого порядка с постоянными комплексными коэффициентами и однородным по порядку дифференцирования символом посвящена работа [9]. В ней получены критерии единственности решения задачи в ряде случаев: как в общем (все корни характеристического уравнения простые и ни один из них не принимает значения $\pm i$ ), так и в случае кратных корней, а также корней, принимающих значения $\pm i$ (называемых ниже исключительными случаями). Отметим, что результаты настоящего исследования посвящены бестипньм уравнениям высокого порядка, однако, результаты работы [9] для правильно эллиптических уравнений вьводятся из теоремы 1 настоящей работы при $m=2$ в общем случае и из результатов работы [10], посвященной получению критериев единственности решения задачи Дирихле для общего уравнения четвертого порядка с постоянными комплексными коэффициентами и однородным символом в исключительньх случаях.

2. Постановка задачи и формулировка результата. В единичном круге $K$ рассматривается следующая задача Дирихле для линейных уравнений произвольного четного порядка $2 m$ с постоянными комплексными коэффициентами и однородным невырожденньм символом:

$$
\begin{gathered}
L(\partial) u=a_{0} \frac{\partial^{2 m} u}{\partial x_{1}^{2 m}}+a_{1} \frac{\partial^{2 m} u}{\partial x_{1}^{2 m-1} \partial x_{2}}+\cdots+a_{2 m-1} \frac{\partial^{2 m} u}{\partial x_{1} \partial x_{2}^{2 m-1}}+a_{2 m} \frac{\partial^{2 m} u}{\partial x_{2}^{2 m}}=0 \\
\left.u\right|_{\partial K}=0,\left.\quad u_{\nu}^{\prime}\right|_{\partial K}=0, \quad \ldots,\left.\quad u_{\nu}^{(m-1)}\right|_{\partial K}=0
\end{gathered}
$$

где $\vec{\nu}$ - единичньй вектор внешней нормали. Заметим, что в силу разложения символа $L(\xi)=a_{0} \xi_{1}^{2 m}+a_{1} \xi_{1}^{2 m-1} \xi_{2}+\cdots+a_{2 m} \xi_{2}^{2 m}=\left\langle\xi, a^{1}\right\rangle\left\langle\xi, a^{2}\right\rangle \cdots\left\langle\xi, a^{2 m}\right\rangle$, где $a^{j} \in \mathbb{C}^{2}$, $j=1,2, \ldots, 2 m,-$ комплексные векторы, $\langle a, b\rangle=a_{1} \bar{b}_{1}+a_{2} \bar{b}_{2}$ для $a, b \in \mathbb{C}^{2}$, уравнение (1) можно записать иначе:

$$
\left\langle\nabla, a^{1}\right\rangle\left\langle\nabla, a^{2}\right\rangle \cdots\left\langle\nabla, a^{2 m}\right\rangle u=0
$$

Наши предположения заключаются в том, что корни $\lambda_{j}$ характеристического полинома $L(1, \lambda)=0$ простые (т.е. символ невырожден или, что то же, уравнение (1) является уравнением главного типа) и $\lambda_{j} \neq \pm i, j=1,2, \ldots, 2 m$.

Углом наклона характеристики, отвечающей некоторому корню $\lambda_{j}$ характеристического уравнения будем называть какое-нибудь решение уравнения $-\operatorname{tg} \varphi_{j}=\lambda_{j}$, $j=1,2, \ldots, 2 m$. Ограничение $\lambda_{j} \neq \pm i, j=1,2, \ldots, 2 m$, как раз связано с тем, что уравнение $\operatorname{tg}(x)= \pm i$ не имеет решений. В дальнейшем будем рассматривать также векторы $\tilde{a}^{j}=\left(-\bar{a}_{2}^{j}, \bar{a}_{1}^{j}\right)=\left(-\cos \varphi_{j}, \sin \varphi_{j}\right), j=1,2, \ldots, 2 m$.

Основным результатом работы является следующая теорема. 
Теорема 1. Для нетривиальной разрешимости задачи (1), (2) в $C^{2 m}(\bar{K})$ необходимо и достаточно, чтобы углы наклона характеристик удовлетворяли следующему условию:

$\operatorname{det}\left(\begin{array}{ccccc}\cos n \varphi_{1} & \sin n \varphi_{1} & \ldots & \cos (n-2(m-1)) \varphi_{1} & \sin (n-2(m-1)) \varphi_{1} \\ \vdots & \vdots & \ddots & \vdots & \vdots \\ \cos n \varphi_{2 m} & \sin n \varphi_{2 m} & \ldots & \cos (n-2(m-1)) \varphi_{2 m} & \sin (n-2(m-1)) \varphi_{2 m}\end{array}\right)=0$

для некоторого $n \in \mathbb{N}, n>2 m-1$. При выполнении этого условия существует полиномиальное решение задачи (1), (2).

В п. 7 приведено простое достаточное условие вьполнения условия (4), состоящее в вещественности и $\pi$-рациональности всех разностей $\varphi_{i}-\varphi_{j}$, откуда, в частности, следует, что ядро оператора (1), (2) бесконечномерно. Приведены также примеры эллиптических (но не правильно эллиптических) скалярных уравнений с таким свойством.

3. Двойственность уравнение-область. Доказательство необходимости в теореме 1 основано на следующем наблюдении, сделанном одним из авторов в работе [11].

Пусть $\Omega \subset \mathbb{R}^{n}$ - ограниченная полуалгебраическая область, заданная неравенством $\Omega=\left\{x \in \mathbb{R}^{n}: P(x)>0\right\}$, с некоторым вещественным полиномом $P$. Уравнение $P(x)=0$ задает границу $\partial \Omega$. Предполагается, что граница области $\Omega$ невырождена: $|\nabla P| \neq 0$ на $\partial \Omega$. Рассматривается краевая задача для уравнения порядка $l$ с постоянньми комплексньми коэффициентами

$$
\left\{\begin{array}{l}
L u=0, \\
\left.u\right|_{\partial \Omega}=0,\left.\quad u_{\nu}^{\prime}\right|_{\partial \Omega}=0, \quad \ldots,\left.\quad u_{\nu}^{(\gamma-1)}\right|_{\partial \Omega}=0,
\end{array}\right.
$$

$\gamma \leqslant l$. Под двойственностью уравнение--область понимается соответствие между задачей (5) и уравнением

$$
P\left(-D_{\xi}\right)^{l-\gamma}\{L(\xi) w(\xi)\}=0,
$$

отмеченное в следующем утверждении.

УТВЕРЖДЕНИЕ 2 ([11]). Для каждого нетривиального решения задачи (5) из $C^{l}(\bar{\Omega})$ существует нетривиальное аналитическое в $\mathbb{C}^{n}$ решение уравнения (6) из некоторого класса $Z$ иелых функиий и наоборот: каждому ненулевому решению $w \in Z$ уравнения (6) отвечает ненулевое решение $u \in C^{l}(\bar{\Omega})$ задачи (5). Класс $Z$ здесь определен как пространство образов Фурье функиий вида $\theta_{\Omega} v, v \in C^{l}\left(\mathbb{R}^{2}\right)$, где $\theta_{\Omega}-$ характеристическая функиия области $\Omega$.

Строгое доказательство этого факта см. в [11]; приведем здесь для удобства читателя наглядные соображения, ведущие к нему. Пусть для простоты $l=2, \gamma=1$, $\tilde{u} \in C^{2}\left(\mathbb{R}^{n}\right)$ - продолжение решения $u$ задачи $(1),(2)$. Применяя к произведению $\tilde{u} \theta_{\Omega}$ оператор $L$, по формуле Лейбница получим выражение вида $A(x) \delta_{\partial \Omega}+l(\nu) u(x)\left(\delta_{\partial \Omega}\right)_{\nu}^{\prime}$, где $A$ зависит от $u, u_{\nu}^{\prime}, u_{\tau}^{\prime}, \tau$ - касательное направление, $\left\langle\delta_{\partial \Omega}, \phi\right\rangle=\int_{\partial \Omega} \bar{\phi}(s) d s$. Учитывая граничное условие $\left.u\right|_{\partial \Omega}=0$, будем иметь $L\left(\tilde{u} \theta_{\Omega}\right)=B(x) \delta_{\partial \Omega}$. Здесь, как и в одномерном анализе, $\psi \delta^{\prime}=-\psi^{\prime} \delta$ при $\psi(0)=0$. Умножая равенство $L\left(\tilde{u} \theta_{\Omega}\right)=B(x) \delta_{\partial \Omega}$ на $P(x)$ и применяя к полученному преобразование Фурье, приходим к искомому равенству (6). Рассуждения можно обратить. 
4. Доказательство необходимости. Предположим, что задача (1), (2) имеет нетривиальное решение в $C^{2 m}(\bar{K})$; тогда согласно утверждению 2 существует нетривиальное аналитическое в $\mathbb{C}^{2}$ решение $w(\xi)$ уравнения

$$
\left(\Delta_{\xi}+1\right)^{m}\{L(\xi) \cdot w(\xi)\}=0
$$

из некоторого класса $Z$ целых функций. Так как функция $w(\xi)$ целая, то функция $v(\xi)=$ $L(\xi) \cdot w(\xi)$ делится на символ $L(\xi)=\left\langle\xi, a^{1}\right\rangle\left\langle\xi, a^{2}\right\rangle \cdots\left\langle\xi, a^{2 m}\right\rangle$ уравнения (3) в классе целых функций; поэтому

$$
\left.v\right|_{\left\langle\xi, a^{1}\right\rangle=0}=0,\left.\quad v\right|_{\left\langle\xi, a^{2}\right\rangle=0}=0, \quad \ldots,\left.\quad v\right|_{\left\langle\xi, a^{2 m}\right\rangle=0}=0 .
$$

Таким образом, получаем двойственную задачу $(7),(8)$ относительно функции $v(\xi)=$ $L(\xi) \cdot w(\xi)$, которая согласно утверждению 2 имеет нетривиальное решение в некотором классе $Z$ целых функций. Раскладывая функцию $v(\xi)$ в степенной ряд, для младшей нетривиальной однородной полиномиальной части ряда $v_{N}(\xi)$ получим задачу

$$
\begin{gathered}
\Delta_{\xi}^{m} v_{N}(\xi)=0, \\
\left.v_{N}\right|_{\left\langle\xi, a^{1}\right\rangle=0}=0,\left.\quad v_{N}\right|_{\left\langle\xi, a^{2}\right\rangle=0}=0, \quad \ldots,\left.\quad v_{N}\right|_{\left\langle\xi, a^{2 m}\right\rangle=0}=0 .
\end{gathered}
$$

Из соотношений (32.15)-(32.22) в [12] или из формулы Альманси на с. 208 в [13] следует, что общее полиномиальное решение уравнения (9) можно записать в виде

$$
\tilde{v}(\xi)=\operatorname{Re}\left\{f_{1}(z)+\cdots+\bar{z}^{m-1} \cdot f_{m}(z)\right\}+i \operatorname{Re}\left\{g_{1}(z)+\cdots+\bar{z}^{m-1} \cdot g_{m}(z)\right\},
$$

где $z=\xi_{1}+i \xi_{2}, f_{i}(z)=\sum f_{i n} \cdot z^{n}, g_{i}(z)=\sum g_{i n} \cdot z^{n}, i=1,2, \ldots, m,-$ некоторые полиномы. Переходя к тригонометрической форме записи комплексного числа $z=\rho \cdot e^{i \varphi}$, получим

$$
\begin{aligned}
\tilde{v}(\rho, \varphi)= & \sum_{n=0}^{\infty} \rho^{n}\left(\alpha_{1 n} \cos n \varphi-\beta_{1 n} \sin n \varphi+\alpha_{2 n} \cos (n-2) \varphi-\beta_{2 n} \sin (n-2) \varphi+\cdots\right. \\
& \left.+\alpha_{m n} \cos (n-2(m-1)) \varphi-\beta_{m n} \sin (n-2(m-1)) \varphi\right) .
\end{aligned}
$$

Здесь постоянные $\alpha_{i n}, \beta_{i n} \in \mathbb{C}$ строятся по коэффициентам разложения полиномов $f_{i}(z)$, $g_{i}(z)$ в степенной ряд следующим образом:

$$
\begin{aligned}
& \operatorname{Re} \alpha_{i n}=\operatorname{Re} f_{i, n-(i-1)}, \quad \operatorname{Im} \alpha_{i n}=\operatorname{Re} g_{i, n-(i-1)}, \\
& \operatorname{Re} \beta_{i n}=\operatorname{Im} f_{i, n-(i-1)}, \quad \operatorname{Im} \beta_{i n}=\operatorname{Im} g_{i, n-(i-1)},
\end{aligned}
$$

Подставляя (11) в (10) и учитывая, что условие $\left\langle\xi, a^{j}\right\rangle=0, j=1, \ldots, 2 m$, эквивалентно равенству $\varphi=-\varphi_{j}$, получим линейную однородную систему алгебраических уравнений относительно постоянных $\alpha_{i n}, \beta_{i n}$

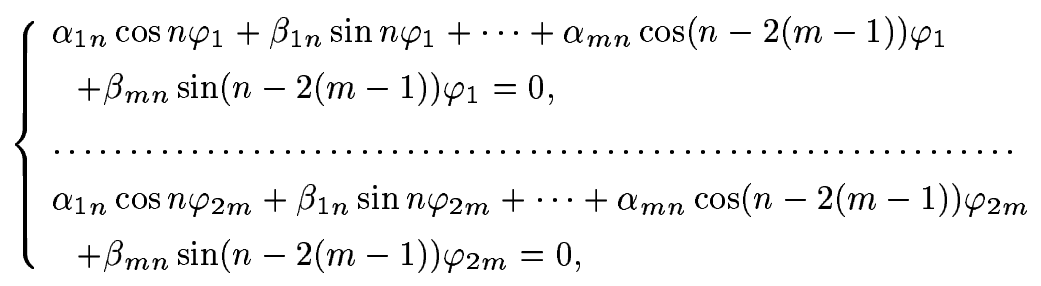


определитель которой стоит в левой части равенства (4). По нашему предположению о существовании нетривиального решения задачи $(1),(2)$ и утверждению 2 , существует нетривиальное решение задачи $(9),(10)$ в классе однородных полиномов некоторой степени $N>2 m-1$, поэтому для такого $N$ существует ненулевой набор $\left(\alpha_{1 N}, \beta_{1 N}, \ldots\right.$, $\left.\alpha_{m N}, \beta_{m N}\right) \in \mathbb{C}^{2 m}$ постоянньх, являющийся решением однородной системы линейных уравнений (12) с $n=N$, что влечет обращение в нуль определителя этой системы. Следовательно, условие (4) выполнено с $n=N$.

5. Доказательство достаточности. При выполнении условия (4) для некоторого $n \in \mathbb{N}, n>2 m-1$, построим нетривиальное решение задачи Дирихле (1), (2) в явном виде. Тем самым будет доказана достаточность условия (4) для нетривиальной разрешимости задачи $(1),(2)$. Ясно, что в силу ортогональности векторов $a^{j}$ и $\tilde{a}^{j}$ при каждом $j=1, \ldots, 2 m$ решением уравнения (1) или (3) является функция

$$
u(x)=\sum_{j=1}^{2 m} C_{j} F_{j}\left(-\tilde{a}^{j} \cdot x\right)
$$

где $F_{j}(y)$ - некоторые гладкие функции одного аргумента, $C_{j}$ - некоторые постоянные, $j=1, \ldots, 2 m$.

Для построения нетривиального решения задачи $(1),(2)$ нам необходимо так подобрать функции $F_{j}\left(-\tilde{a}^{j} \cdot x\right)$, чтобы функция вида (13) удовлетворяла граничным условиям (2) для некоторого ненулевого набора $\widetilde{C}=\left(\widetilde{C}_{1}, \ldots, \widetilde{C}_{2 m}\right)$ постоянных.

Оказалось (доказательство см. ниже), что если в качестве функций $F_{j}(y)$ взять одну и ту же функцию

$$
\begin{gathered}
F_{j}(y)=F_{m-1}(y):=\int_{0}^{y} d y_{1} \int_{0}^{y_{1}} d y_{2} \cdots \int_{0}^{y_{m-3}} d y_{m-2} \int_{0}^{y_{m-2}} T_{N}(t) \cdot d t \\
j=1, \ldots, 2 m,
\end{gathered}
$$

где $T_{N}(t)$ - полином Чебьшева первого рода порядка $N=n-m+1$, а в качестве ненулевого набора $\widetilde{C}=\left(\widetilde{C}_{1}, \ldots, \widetilde{C}_{2 m}\right)$ взять некоторое нетривиальное решение $C^{*}=\left(C_{1}^{*}, \ldots\right.$, $C_{2 m}^{*}$ ) линейной алгебраической системы с определителем, стоящим в левой части равенства (4), то функция $u(x)$ из $(13)$ с $C_{j}=C_{j}^{*}$ будет являться искомым нетривиальным решением однородной задачи Дирихле (1), (2).

Приступая к доказательству, мы проверим сначала, что интеграл (14) можно записать в виде

$$
F_{m-1}(y)= \begin{cases}\sum_{k=0}^{l}\left\{C_{k}^{+}(N) T_{N+2 k+1}(y)+C_{k}^{-}(N) T_{N-2 k-1}(y)\right\} & \text { при } m-1=2 l+1, \\ \sum_{k=0}^{l}\left\{D_{k}^{+}(N) T_{N+2 k}(y)+D_{k}^{-}(N) T_{N-2 k}(y)\right\} & \text { при } m-1=2 l .\end{cases}
$$

Соотношения (15) нетрудно доказать индукцией по $N$ на основе следующего равенства:

$$
\int_{0}^{x} T_{N}(t) d t=\frac{1}{2(N+1)} T_{N+1}(x)-\frac{1}{2(N-1)} T_{N-1}(x),
$$


которое нетрудно получить из соотношения (37) при $m=1$ на с. 187 в [14] и (2) на с. 185 там же, и которое означает равенство (15) при $m=2$. Здесь

$$
C_{0}^{+}(N)=\frac{1}{2(N+1)}, \quad C_{0}^{-}(N)=\frac{1}{2(N-1)} .
$$

Из равенств (15) следует, что (13) представимо следующим образом:

$$
u(x)=\left\{\begin{array}{l}
\sum_{j=1}^{2 m} C_{j} \sum_{k=0}^{l}\left\{C_{k}^{+}(N) T_{N+2 k+1}\left(-\tilde{a}^{j} \cdot x\right)+C_{k}^{-}(N) T_{N-2 k-1}\left(-\tilde{a}^{j} \cdot x\right)\right\} \\
\quad \text { при } m-1=2 l+1, \\
\sum_{j=1}^{2 m} C_{j} \sum_{k=0}^{l}\left\{D_{k}^{+}(N) T_{N+2 k}\left(-\tilde{a}^{j} \cdot x\right)+D_{k}^{-}(N) T_{N-2 k}\left(-\tilde{a}^{j} \cdot x\right)\right\} \\
\quad \text { при } m-1=2 l,
\end{array}\right.
$$

где $C_{j}$ - произвольные постоянные, $C_{k}^{+}(N), C_{k}^{-}(N), D_{k}^{+}(N), D_{k}^{-}(N)$ - постоянные, зависящие только от $N$ и возникающие при вычислении интегралов в (14). Здесь порядок многочлена $u(x)$ равен $n$ при $N=n-m+1$.

Покажем, что функция (16) удовлетворяет граничным условиям $(2)$, если $C_{j}=C_{j}^{*}-$ некоторое нетривиальное решение линейной однородной системы с определителем (4). Для определенности рассмотрим случай четного $m: m-1=2 l+1$. Учитьвая, что на $\partial K\left(\tilde{a}^{j} \cdot x\right)=-\cos \left(\tau+\varphi_{j}\right)$, a $T_{N}\left(\cos \left(\tau+\varphi_{j}\right)\right)=\cos N\left(\tau+\varphi_{j}\right)$, будем иметь

$$
\begin{aligned}
\left.u\right|_{\partial K}= & \sum_{j=1}^{2 m} C_{j} \sum_{k=0}^{l}\left\{C_{k}^{+}(N) \cos (N+2 k+1)\left(\tau+\varphi_{j}\right)+C_{k}^{-}(N) \cos (N-2 k-1)\left(\tau+\varphi_{j}\right)\right\} \\
= & \sum_{k=0}^{l} C_{k}^{+}(N) \cos (N+2 k+1) \tau \sum_{j=1}^{2 m} C_{j} \cos (N+2 k+1) \varphi_{j} \\
& -\sum_{k=0}^{l} C_{k}^{+}(N) \sin (N+2 k+1) \tau \sum_{j=1}^{2 m} C_{j} \sin (N+2 k+1) \varphi_{j} \\
& +\sum_{k=0}^{l} C_{k}^{-}(N) \cos (N-2 k-1) \tau \sum_{j=1}^{2 m} C_{j} \cos (N-2 k-1) \varphi_{j} \\
& -\sum_{k=0}^{l} C_{k}^{-}(N) \sin (N-2 k-1) \tau \sum_{j=1}^{2 m} C_{j} \sin (N-2 k-1) \varphi_{j}=0 .
\end{aligned}
$$

Последнее равенство справедливо для всех $\tau$ тогда и только тогда, когда имеют место следуюшие соотношения:

$$
\begin{cases}\sum_{j=1}^{2 m} C_{j} \cos (N+2 k+1) \varphi_{j}=0, & \sum_{j=1}^{2 m} C_{j} \cos (N-2 k-1) \varphi_{j}=0 \\ \sum_{j=1}^{2 m} C_{j} \sin (N-2 k-1) \varphi_{j}=0, & \sum_{j=1}^{2 m} C_{j} \sin (N+2 k+1) \varphi_{j}=0\end{cases}
$$

при каждом $k=0,1, \ldots, l$. Соотношения (17) представляют собой систему $2 m$ уравнений с $2 m$ неизвестными, определитель которой равен нулю в силу условия (4) (если 
положить $N+m-1=n)$. Следовательно, если $C_{j}=C_{j}^{*}, j=1, \ldots, 2 m,-$ решение системы $(17)$, то функция $u(x)=\sum_{j=1}^{2 m} C_{j}^{*} F_{m-1}\left(-\tilde{a}^{j} \cdot x\right)$ удовлетворяет первому граничному условию в (2).

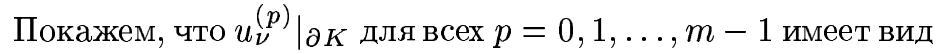

$$
\begin{aligned}
\left.u_{\nu}^{(p)}\right|_{\partial K}= & \sum_{j=1}^{2 m} C_{j} \sum_{k=0}^{l}\left\{P_{k}^{+}(N, p) \cos (N+2 k+1)\left(\tau+\varphi_{j}\right)\right. \\
& \left.+P_{k}^{-}(N, p) \cos (N-2 k-1)\left(\tau+\varphi_{j}\right)\right\}
\end{aligned}
$$

с некоторыми постоянными, поэтому равенство $\left.u_{\nu}^{(p)}\right|_{\partial K}=0$ снова приведет нас к тому, что постоянные $C_{1}, C_{2}, \ldots, C_{2 m}$ достаточно подчинить системе (17). Тем самьм, теорема 1 будет полностью доказана.

Действительно,

$$
\begin{aligned}
\left.u_{\nu}^{(p)}\right|_{\partial K} & =\sum_{j=1}^{2 m} C_{j} \cos ^{p}\left(\tau+\varphi_{j}\right) F_{m-1}^{(p)}\left(\cos \left(\tau+\varphi_{j}\right)\right) \\
& =\sum_{j=1}^{2 m} C_{j} \cos ^{p}\left(\tau+\varphi_{j}\right) F_{m-p-1}\left(\cos \left(\tau+\varphi_{j}\right)\right) .
\end{aligned}
$$

Для определенности будем считать $p$ четным: $p=2 q$; тогда $m-p-1=2 l+1-2 q=$ $2(l-q)+1$ нечетное и

$$
\cos ^{p}\left(\tau+\varphi_{j}\right)=\sum_{i=0}^{q} \alpha_{2 i}^{(2 q)} \cos 2 i\left(\tau+\varphi_{j}\right)
$$

Коэффициенты $\alpha_{2 i}^{(2 q)}$ определяются из следуюших рекуррентных соотношений: $\alpha_{1}^{(1)}=1$, $\alpha_{0}^{(2)}=\alpha_{2}^{(2)}=1 / 2, \alpha_{0}^{(2 q)}=\frac{1}{2} \alpha_{1}^{(2 q-1)}, \alpha_{2 q}^{(2 q)}=\frac{1}{2} \alpha_{2 q-1}^{(2 q-1)}, \alpha_{2 i}^{(2 q)}=\frac{1}{2} \alpha_{2 i-1}^{(2 q-1)}+\frac{1}{2} \alpha_{2 i+1}^{(2 q-1)}$, $i=1,2, \ldots, q-1$. С учетом соотношений (20) и (15) равенство (19) примет вид

$$
\begin{aligned}
\left.u_{\nu}^{(p)}\right|_{\partial K}= & \sum_{j=1}^{2 m} C_{j} \sum_{i=0}^{q} \alpha_{2 i}^{(2 q)} \cos 2 i\left(\tau+\varphi_{j}\right) \sum_{k=0}^{l-q}\left\{C_{k}^{+}(N) \cos (N+2 k+1)\left(\tau+\varphi_{j}\right)\right. \\
& \left.\quad+C_{k}^{-}(N) \cos (N-2 k-1)\left(\tau+\varphi_{j}\right)\right\} \\
= & \frac{1}{2} \sum_{j=1}^{2 m} C_{j} \sum_{i=0}^{q} \alpha_{2 i}^{(2 q)} \sum_{k=0}^{l-q}\left\{C _ { k } ^ { + } ( N ) \left(\cos (N+2(k+i)+1)\left(\tau+\varphi_{j}\right)\right.\right. \\
& \left.\quad+\cos (N+2(k-i)+1)\left(\tau+\varphi_{j}\right)\right) \\
& +C_{k}^{-}(N)\left(\cos (N-2(k-i)-1)\left(\tau+\varphi_{j}\right)\right. \\
& \left.\left.\quad+\cos (N-2(k+i)-1)\left(\tau+\varphi_{j}\right)\right)\right\} \\
= & \sum_{j=1}^{2 m} C_{j} \sum_{k=0}^{l}\left\{P_{k}^{+}(N, p) \cos (N+2 k+1)\left(\tau+\varphi_{j}\right)\right. \\
& \left.\quad+P_{k}^{-}(N, p) \cos (N-2 k-1)\left(\tau+\varphi_{j}\right)\right\}
\end{aligned}
$$

Случай нечетного $p$ аналогичен. Теорема доказана. 
6. Случаи уравнений 2-, 4- и 6-го порядков. 1) Рассмотрим однородную задачу Дирихле в круге для уравнения второго порядка, при этом как в настоящем примере, так и в нижеследуюших, наши предположения на коэффициенты и символ уравнения из п. 2 остаются в силе:

$$
a_{0} \frac{\partial^{2} u}{\partial x_{1}^{2}}+a_{1} \frac{\partial^{2} u}{\partial x_{1} \partial x_{2}}+a_{2} \frac{\partial^{2} u}{\partial x_{2}^{2}}=0,\left.\quad u\right|_{\partial K}=0 .
$$

В этом случае $m=1$, поэтому необходимьм и достаточным условием нетривиальной разрешимости задачи (19) является выполнение условия

$$
\operatorname{det}\left(\begin{array}{cc}
\cos n \varphi_{1} & \sin n \varphi_{1} \\
\cos n \varphi_{2} & \sin n \varphi_{2}
\end{array}\right)=0
$$

для некоторого $n \in \mathbb{N}, n>0$. Очевидно, что условие (22) эквивалентно $\pi$-рациональности угла между характеристиками. В силу (16) ненулевое решение задачи (21) можно записать в виде

$$
u(x)=C_{1}^{*} T_{n}\left(-\tilde{a}^{1} \cdot x\right)+C_{2}^{*} T_{n}\left(-\tilde{a}^{2} \cdot x\right),
$$

где $\left(C_{1}^{*}, C_{2}^{*}\right)$ - решение линейной однородной системы, определитель которой удовлетворяет условию (22). Отметим, что этот результат был получен в работе [8] другим методом.

$2)$ В случае уравнения четвертого порядка $(m=2)$ критерием существования нетривиального решения задачи Дирихле в круге служит выполнение равенства

$$
\operatorname{det}\left(\begin{array}{cccc}
\cos n \varphi_{1} & \sin n \varphi_{1} & \cos (n-2) \varphi_{1} & \sin (n-2) \varphi_{1} \\
\vdots & \vdots & \vdots & \vdots \\
\cos n \varphi_{4} & \sin n \varphi_{4} & \cos (n-2) \varphi_{4} & \sin (n-2) \varphi_{4}
\end{array}\right)=0
$$

для некоторого $n \in \mathbb{N}, n>2$. А ненулевое решение задачи

$$
\begin{gathered}
a_{0} \frac{\partial^{4} u}{\partial x_{1}^{4}}+a_{1} \frac{\partial^{4} u}{\partial x_{1}^{3} \partial x_{2}}+a_{2} \frac{\partial^{4} u}{\partial x_{1}^{2} \partial x_{2}^{2}}+a_{3} \frac{\partial^{4} u}{\partial x_{1} \partial x_{2}^{3}}+a_{4} \frac{\partial^{4} u}{\partial x_{2}^{4}}=0 \\
\left.u\right|_{\partial K}=0,\left.\quad u_{\nu}^{\prime}\right|_{\partial K}=0
\end{gathered}
$$

имеет вид

$$
u(x)=\sum_{j=1}^{4} C_{j}^{*}\left(\frac{1}{2 n} T_{n}\left(-\tilde{a}^{j} \cdot x\right)-\frac{1}{2(n-2)} T_{n-2}\left(-\tilde{a}^{j} \cdot x\right)\right) .
$$

Этот результат был получен в работе [15] без использования метода двойственности уравнение-область.

3) Для уравнения шестого порядка $(m=3)$ условие $(4)$ примет вид

$$
\operatorname{det}\left(\begin{array}{cccccc}
\cos n \varphi_{1} & \sin n \varphi_{1} & \cos (n-2) \varphi_{1} & \sin (n-2) \varphi_{1} & \ldots & \sin (n-4) \varphi_{1} \\
\vdots & \vdots & \vdots & \vdots & \vdots & \vdots \\
\cos n \varphi_{6} & \sin n \varphi_{6} & \cos (n-2) \varphi_{6} & \sin (n-2) \varphi_{6} & \ldots & \sin (n-4) \varphi_{6}
\end{array}\right)=0
$$


для некоторого $n \in \mathbb{N}, n>4$, а нетривиальное решение соответствующей задачи можно записать следующим образом:

$$
\begin{aligned}
u(x)= & \sum_{j=1}^{6} C_{j}^{*}\left(\frac{1}{4 n(n-1)} T_{n}\left(-\tilde{a}^{j} \cdot x\right)-\frac{1}{2(n-3)(n-1)} T_{n-2}\left(-\tilde{a}^{j} \cdot x\right)\right. \\
& \left.+\frac{1}{4(n-3)(n-4)} T_{n-4}\left(-\tilde{a}^{j} \cdot x\right)\right)
\end{aligned}
$$

Здесь, как и прежде, $\left(C_{1}^{*}, C_{2}^{*}, \ldots, C_{6}^{*}\right)$ - некоторое решение однородной системы, определитель которой удовлетворяет условию (24).

7. Примеры. Приведем некоторые достаточные условия на коэффициенты оператора исходного уравнения, вьполнение которых повлечет за собой справедливость равенства (4). В случае $m=2$ эти условия окажутся полезными при построении примеров вещественных неправильно эллиптических систем четвертого порядка, сводящихся к одиночным уравнениям с постоянными комплексными коэффициентами, для которых однородная задача Дирихле в круге имеет счетное число линейно независимых полиномиальных решений.

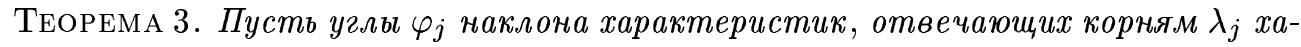
рактеристического уравнения, таковьи, что $\varphi_{i}-\varphi_{j} \in \pi \mathbb{Q}, i \neq j, i, j=1,2, \ldots, 2 m$, т.е. все разности $\varphi_{i}-\varphi_{j}$ вещественны и т-рациональны. Тогда однородная задача Дирихле в круге для соответствуюшего дифференииального уравнения будет иметь счетное число линейно независимых полиномиальных решений.

ДокАЗАТЕЛЬСтво. Действительно, если $\varphi_{i}-\varphi_{j}=\pi k_{i j} / n_{i j}$ для некоторых целых $k_{i j}, n_{i j}, i, j=1,2, \ldots, 2 m$, то полагая $n$ равным наименьшему общему кратному чисел $n_{i j}, i, j=1,2, \ldots, 2 m$, получим справедливость нашего утверждения, раскладывая определитель в равенстве (4) по первым двум столбцам. Заметим, что, если $\varphi_{i}-\varphi_{j}=\pi k_{i j} / N$, что влечет выполнение равенства (4) с $n=N$, то, очевидно, $\varphi_{i}-\varphi_{j}=\pi l k_{i j} / l N$, что влечет справедливость равенства (4) с $n=l N$. Отсюда, используя достаточность в утверждении теоремы 1 , получим наличие нетривиального решения однородной задачи Дирихле в круге в виде полинома степени $l N$ с любьм $l \in \mathbb{N}$. Тем самьм, теорема 3 доказана.

ЗАмЕчАниЕ 1 . Отметим, что если уравнение (1) является правильно эллиптическим, то все разности $\varphi_{i}-\varphi_{j}, i, j=1,2, \ldots, 2 m$, не могут быть вещественньми, поскольку в противном случае корни $\tau$ уравнения $L(\xi+\tau \eta)=0$ не были бы равномерно распределены в верхней и нижней Im-полуплоскости (см. [3, с. 134]). Следовательно, уравнения, описываемые в теореме 3 , не являются правильно эллиптическими и для них (согласно теореме 3) имеет место бесконечномерная неединственность решения однородной задачи Дирихле в круге.

Обратимся к примерам. Рассмотрим случай $m=2$. В нижеследующих вычислениях нам будет удобнее использовать углы наклона бихарактеристик $\psi_{j}$, связанные с углами наклона характеристик $\varphi_{j}$ очевидными равенствами: $\psi_{j}+\varphi_{j}=\pi / 2, j=1, \ldots, 2 m$. Пусть углы наклона бихарактеристик $\psi_{j}=x_{j}+i y_{j}$, и параметры $b_{j}, s_{j} \in \mathbb{R}, s_{j} \leqslant 1$, $b_{j}>0, j=1,2,3,4$, выбраны для удобства вычислений следуюшим образом: $\sin x_{j}=s_{j}$, 
$y_{j}=\ln \sqrt{b}_{j}, j=1,2,3,4$. Тогда из соотношений $\lambda_{j}=\operatorname{ctg} \psi_{j}, j=1,2,3,4$, будет следовать

$$
\lambda_{j}=\frac{4 b_{j} s_{j} \sqrt{1-s_{j}^{2}}}{4 b_{j} s_{j}^{2}+\left(b_{j}-1\right)^{2}}-i \frac{\left(b_{j}^{2}-1\right)}{4 b_{j} s_{j}^{2}+\left(b_{j}-1\right)^{2}}, \quad j=1,2,3,4
$$

Согласно утверждению теоремы 3 , параметры $s_{j}, b_{j}, j=1,2,3,4$, следует подбирать так, чтобы

$$
\arcsin s_{i}-\arcsin s_{j}=\frac{\pi k_{i j}}{n}, \quad b_{i}=b_{j}, \quad \forall i \neq j, \quad i, j=1,2,3,4 .
$$

Тогда параметрическое семейство (25) корней характеристического уравнения определяет параметрическое семейство вещественньх эллиптических систем четвертого порядка с указанньм выше свойством.

Например, соотношениям (26) при $n=4$ удовлетворяют следующие наборы углов:
a) $\psi_{1}=\pi+\frac{i}{4} \ln 3, \quad \psi_{2}=\frac{\pi}{4}+\frac{i}{4} \ln 3, \quad \psi_{3}=\frac{\pi}{2}+\frac{i}{4} \ln 3, \quad \psi_{4}=-\frac{\pi}{4}+\frac{i}{4} \ln 3$,
b) $\psi_{1}=\pi+\frac{i}{4} \ln 5, \quad \psi_{2}=\frac{\pi}{4}+\frac{i}{4} \ln 5, \quad \psi_{3}=\frac{\pi}{2}+\frac{i}{4} \ln 5, \quad \psi_{4}=-\frac{\pi}{4}+\frac{i}{4} \ln 5$.

Им отвечают уравнения
a) $\frac{\partial^{4} u}{\partial x_{1}^{4}}+5 i \frac{\partial^{4} u}{\partial x_{1}^{3} \partial x_{2}}-6 \frac{\partial^{4} u}{\partial x_{1}^{2} \partial x_{2}^{2}}-5 i \frac{\partial^{4} u}{\partial x_{1} \partial x_{2}^{3}}+\frac{\partial^{4} u}{\partial x_{2}^{4}}=0$,
b) $3 \frac{\partial^{4} u}{\partial x_{1}^{4}}+13 i \frac{\partial^{4} u}{\partial x_{1}^{3} \partial x_{2}}-18 \frac{\partial^{4} u}{\partial x_{1}^{2} \partial x_{2}^{2}}-13 i \frac{\partial^{4} u}{\partial x_{1} \partial x_{2}^{3}}+3 \frac{\partial^{4} u}{\partial x_{2}^{4}}=0$,

которые являются эллиптическими, поскольку их символы не имеют вещественных корней и не являются правильно эллиптическими в силу замечания 2 . А соответствующие вещественные системы, для которых однородная задача Дирихле в круге будет иметь счетное число линейно независимых полиномиальных решений, записываются следующим образом:
a) $\left\{\begin{array}{l}\frac{\partial^{4} v}{\partial x_{1}^{4}}-5 \frac{\partial^{4} w}{\partial x_{1}^{3} \partial x_{2}}-6 \frac{\partial^{4} v}{\partial x_{1}^{2} \partial x_{2}^{2}}+5 \frac{\partial^{4} w}{\partial x_{1} \partial x_{2}^{3}}+\frac{\partial^{4} v}{\partial x_{2}^{4}}=0 \\ \frac{\partial^{4} w}{\partial x_{1}^{4}}+5 \frac{\partial^{4} v}{\partial x_{1}^{3} \partial x_{2}}-6 \frac{\partial^{4} w}{\partial x_{1}^{2} \partial x_{2}^{2}}-5 \frac{\partial^{4} v}{\partial x_{1} \partial x_{2}^{3}}+\frac{\partial^{4} w}{\partial x_{2}^{4}}=0\end{array}\right.$
b) $\left\{\begin{array}{l}3 \frac{\partial^{4} v}{\partial x_{1}^{4}}-13 \frac{\partial^{4} w}{\partial x_{1}^{3} \partial x_{2}}-18 \frac{\partial^{4} v}{\partial x_{1}^{2} \partial x_{2}^{2}}+13 \frac{\partial^{4} w}{\partial x_{1} \partial x_{2}^{3}}+3 \frac{\partial^{4} v}{\partial x_{2}^{4}}=0 \\ 3 \frac{\partial^{4} w}{\partial x_{1}^{4}}+13 \frac{\partial^{4} v}{\partial x_{1}^{3} \partial x_{2}}-18 \frac{\partial^{4} w}{\partial x_{1}^{2} \partial x_{2}^{2}}-13 \frac{\partial^{4} v}{\partial x_{1} \partial x_{2}^{3}}+3 \frac{\partial^{4} w}{\partial x_{2}^{4}}=0\end{array}\right.$ 


\section{СПИСОК ЦИТИРОВАННОЙ ЛИТЕРАТУРЫ}

[1] Бицадзе А. В. О единственности решения задачи Дирихле для эллиптических уравнений с частными производными // УМН. 1948. Т. 3. №6. С. 211-212.

[2] Бицадзе А. В. Уравнения смешанного типа. М.: Физматгиз, 1959.

[3] Лионс ЖК.-Л., Мадженес Э. Неоднородные граничные задачи и их приложения. Т. 1. М.: Мир, 1971.

[4] Марковский А. И. Об области значения эллиптического полинома // Укр. матем. ж. 1973. T. 25. № 2. С. 228-234.

[5] Вишик М.И. О сильно эллиптических системах дифференциальных уравнений // Матем. сб. 1951. Т. 29. №3. С. 615-676.

[6] Александрян Р. А. О задаче Дирихле для уравнения струны и о полноте одной системы функций в круге // Докл. АН СССР. 1950. Т. 73. № 5 .

[7] Александрян Р. А. Спектральные свойства операторов, порожденных системами дифференциальных уравнений типа С. Л. Соболева // Тр. ММО. 1960. Т. 9. С. 455-507.

[8] Бурский В. П. О нарушении единственности решения задачи Дирихле для эллиптических систем в круге // Матем. заметки. 1990. Т. 48. № 3. С. 32-36.

[9] Babayan A. O. On unique solvability of Dirichlet problem for fourth order properly elliptic equation // Izv. Nat. AN Armenii. Matem. 1999. V. 34. № 5. P. 5-18.

[10] Буряченко Е. А. О единственности решений задачи Дирихле в круге для дифференциальных уравнений четвертого порядка в вырожденных случаях // Нелинейные граничные задачи. 2000. T. 10. C. 44-49.

[11] Бурский В.П. О единственности решения некоторых граничных задач для дифференциальных уравнений в области с алгебраической границей // Укр. матем. ж. 1993. Т. 45. № 7. C. $898-906$.

[12] Векуа И.Н. Новые методы решения эллиптических уравнений. М.: ОГИЗ, 1948.

[13] Бицадзе А. В. Некоторые классы дифференциальных уравнений в частных производных. М.: Мир, 1981.

[14] Бейтмен Г., Эрдейи А. Высшие трансцендентные функции. Т. 2. М.: Наука, 1974.

[15] Буряченко Е. А. К вопросу о нарушении единственности решения задачи Дирихле для уравнений с частными производньми четвертого порядка // Тр. ИПММ НАН Украины. 1999. T. 4. C. $15-21$.

Институт прикладной математики и механики НАН Украины

Поступило

E-mail: buryachenko@yahoo.com, burskii@iamm.ac.donetsk.ua

10.01 .2000

Исправленный вариант

09.02.2004 